\title{
Electrochemical enzyme-linked immunosorbent assay (e-ELISA) for parasitic nematode: Ostertagia ostertagi (brown stomach worm) infections in dairy cattle
}

\author{
Baljit Singh \\ Technological University Dublin, baljit.singh@tudublin.ie \\ Evangelia Flampouri \\ Technological University Dublin \\ Eithne Dempsey \\ National University of Ireland, Maynooth
}

Follow this and additional works at: https://arrow.tudublin.ie/ittsciart

Part of the Chemistry Commons

\section{Recommended Citation}

Singh B , Flampouri E, Dempsey E . Electrochemical enzyme-linked immunosorbent assay (e-ELISA) for parasitic nematode Ostertagia ostertagi (brown stomach worm) infections in dairy cattle. Analyst. 2019 Sep 23;144(19):5748-5754. doi: 10.1039/c9an00982e. PMID: 31432061.

This Article is brought to you for free and open access by the School of Science and Computing at ARROW@TU Dublin. It has been accepted for inclusion in Articles by an authorized administrator of ARROW@TU Dublin. For more information, please contact

arrow.admin@tudublin.ie, aisling.coyne@tudublin.ie, gerard.connolly@tudublin.ie.

Funder: Enterprise Ireland; European Regional

Development Fund

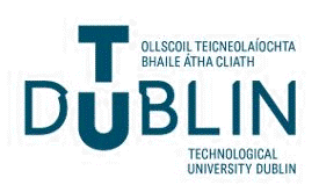


A) Check for updates

Cite this: Analyst, 2019, 144, 5748

\title{
Electrochemical enzyme-linked immunosorbent assay (e-ELISA) for parasitic nematode Ostertagia ostertagi (brown stomach worm) infections in dairy cattle
}

\author{
Baljit Singh, (D) *a Evangelia Flampouri ${ }^{\mathrm{a}}$ and Eithne Dempsey (D) $^{\mathrm{b}}$
}

A sensitive electrochemical immunoassay (e-ELISA) has been developed for the detection of the gastrointestinal parasitic nematode Ostertagia ostertagi (brown stomach worm) in infected and control serum samples. An antigen-indirect immunoassay format was employed to detect the presence of $O$. ostertagi antibodies, coupled with an anti-species monoclonal horseradish peroxidase (HRP) conjugate. ABTS (2,2'-azino-bis(3-ethylbenzothiazoline-6-sulphonic acid)) and TMB (3,3',5,5'-tetramethylbenzidine/ hydrogen peroxide) were investigated as both chromogenic visualising reagents for optical ELISA and electroactive substrates for electrochemical ELISA in the HRP catalysed oxidation reaction. Coulometry was applied for the detection of $O$. ostertagi antibodies (via TMB electrochemistry) and compared with the commercial optical ELISA (ABTS based SVANOVIR® O. ostertagi-Ab ELISA kit). Cost-effective inhouse sensors were designed and fabricated using polyester and chemical adhesive materials with the aid of stencil printing and laser machining techniques. The performance of the electrochemical ELISA and sensor was evaluated by investigating redox mediators (ABTS vs. TMB), stop solutions (sodium dodecyl sulfate vs. sulfuric acid) and incubation times (150 min vs. $70 \mathrm{~min}$ vs. $25 \mathrm{~min}$ ). For a total assay incubation time of 70 minutes, the $\mathrm{TMB} / \mathrm{H}_{2} \mathrm{SO}_{4}$ based e-ELISA was able to differentiate between positive (P) and negative $(\mathrm{N})$ control serum samples, with a $\mathrm{P} / \mathrm{N}_{70}$ control ratio 1.6 times higher than that of optical ELISA ( $\mathrm{TMB} / \mathrm{H}_{2} \mathrm{SO}_{4}$ combination) and 2.9 times higher than that of the commercial ELISA kit (ABTS/SDS combination). Furthermore, the e-ELISA approach is quicker and required only $25 \mathrm{~min}$ (total incubation time) with even better response ( $\left.P / N_{25}=14.7\right)$, which is approximately 4 -fold higher than the optical immunoassay $\left(P / N_{25}=3.8\right)$. The proposed e-ELISA is specific (selective Ab-Ag interactions) and highly sensitive capable of detecting up to 16 -fold dilutions of a positive control serum sample. The electrochemical ELISA approach has the potential for rapid sample screening in a portable, disposable format, contributing to the quest for effective prevention and control of parasitic Ostertagia ostertagi infections in cattle.

Received 29th May 2019, Accepted 12th August 2019 DOI: 10.1039/c9an00982e rsc.li/analyst

\section{Introduction}

Parasitic gastroenteritis in dairy herds results predominantly from Ostertagia ostertagi infections (ostertagiasis). This abomasal parasitic nematode (round worm) of cattle, also known as medium stomach worm or brown stomach worm, is prevalent in most temperate climates and is naturally transmitted to animals grazing pasture contaminated with larval worms. After ingestion, infective larvae mature to the adult stage, cling to the intestinal lining of the animal and feed on the tissue. Adult worms produce thousands of eggs every day, which are

\footnotetext{
${ }^{a}$ MiCRA Biodiagnostics Technology Gateway, Technological University Dublin (TU Dublin), Tallaght, Dublin 24, D24 FKT9, Ireland. E-mail: Baljit.Singh@TUDublin.ie, Singh.Baljit765@gmail.com; Fax: +353 (01) 404 2753; Tel: +353 (01) 4042033

${ }^{b}$ Department of Chemistry, Maynooth University, Maynooth, Co. Kildare, Ireland
}

released through the animal faeces onto the pasture, maintaining the chain of infection. ${ }^{1}$

Clinical symptoms of ostertagiasis may include diarrhoea, weight loss, malnutrition and even death. The disease is usually chronic and leads to hidden, undiagnosed subclinical losses, such as reduced weight, milk yields and poor fertility rates. $^{2}$ Gastrointestinal nematode infections have considerable negative impact on grass-based production systems, with the EU livestock industry reporting annual losses of $€ 2.3$ billion in dairy cattle and $€ 1.1$ billion in sheep meat production. ${ }^{3}$

Conventional parasitological methods for helminth infections are based on counting eggs or larvae in the animal faeces (faecal egg counts) or on blood analysis for serum pepsinogen activity. ${ }^{4}$ However, neither of these methods has been shown to provide quantitative measures of worm burden and often fail to detect the prepatent stages of the infections or 
assist in the decision for timely and effective application of antihelmintic treatments. ${ }^{5}$ Therefore, it is necessary to establish sensitive, rapid yet reliable field-deployable techniques to identify the early stages of infection at the farm level.

In the last few decades, enzyme-linked immunosorbent assays (ELISAs) have proven to be attractive options for detecting or monitoring infection status in dairy herd health and production management. ${ }^{6}$ ELISAs provide simple, relatively inexpensive, and rapid diagnostic tests for assessing various pathogens. ${ }^{6}$ SVANOVIR® O. ostertagi-Ab (Boehringer Ingelheim Svanova, Uppsala, Sweden) is a commercially available ELISA kit, used in Europe to determine levels of $O$. ostertagi parasitism in milk samples. The kit is an indirect ELISA that measures the levels of $O$. ostertagi antibodies (semi-quantitatively) present in milk or plasma samples. The assay principle is based on a horseradish peroxidase (HRP)-conjugated antibody and a chromogenic substrate, while results are indicated by measuring the optical density with the use of a spectrophotometer. $^{7}$

Electrochemical methods have been successfully combined with immunoassays for frequent and on-site analysis, ${ }^{8}$ with promising results, owing to the operational simplicity, accuracy and sensitivity of electrochemical analysis. ${ }^{9}$ Stencil/ screen-printed electrodes (SPEs) have been widely employed in electrochemical immunosensor/biosensor developments ${ }^{10-13}$ as they obliterate the need for complex instrumentation and provide advantages in terms of cost, portability and mass production capabilities. ${ }^{14,15}$

In the present work, we report a simple and sensitive electronic ELISA (e-ELISA) approach for the rapid determination of O. ostertagi antibodies in serum samples. The use of electrochemical immunoassays in relation to veterinary diagnostics is very limited and any development which expands the Ostertagia ostertagi detection format and leads towards a more portable device with comparable sensitivity and performance is highly desired and will be a significant improvement in this field. The work presented addresses an unmet need in relation to advancement of detection methodologies for this highly significant animal disease. The proposed method employs an in-house fabricated electrochemical sensor for measuring O. ostertagi antibodies through the anti-bovine IgG-HRP-catalysed oxidation of TMB, in the presence of hydrogen peroxide. To the best of our knowledge, this is the first study describing an HRP-labelled electrochemical immunoassay (e-ELISA) that utilises coulometric detection of $O$. ostertagi antibodies for assaying levels of brown stomach worm parasitism.

\section{Experimental}

\section{Materials and reagents}

The SVANOVIR ${ }^{\circledR}$ O. ostertagi-Ab ELISA kit was purchased from Svanova Biotech AB (Boehringer Ingelheim Svanova). PBS-Tween solution $(20 \times)$ was provided with the kit. Biosensorgrade carbon ink was supplied by the Gwent Group (United Kingdom). TMB (3,3',5,5'-tetramethylbenzidine), $\mathrm{H}_{2} \mathrm{O}_{2}$ (hydro- gen peroxide), sulfuric acid $\left(\mathrm{H}_{2} \mathrm{SO}_{4}\right)$ and all other reagents, unless otherwise specified, were purchased from SigmaAldrich (Arklow, Co. Wicklow, Ireland). Scotch Magic ${ }^{\mathrm{TM}}$ tape adhesive material was purchased from $3 \mathrm{M}$ and polyester materials were purchased from DuPont Teijin Films (USA) for sensor fabrication/construction.

\section{Electrochemical sensor fabrication}

Sensors were fabricated using stencil printing and laser machining techniques. Carbon tracks (5 mm wide with a separation of $0.5 \mathrm{~mm}$ ) were stencil printed (manually) on a Melinex white polyester base material (200 $\mu \mathrm{m}$, thickness) using commercial carbon ink (Gwent) and $3 \mathrm{M}$ Scotch Magic ${ }^{\mathrm{TM}}$ tape. Printed carbon tracks (base layer) were allowed to dry at room temperature followed by drying at $60{ }^{\circ} \mathrm{C}$ for 2 hours before the application of an insulation layer. The insulation layer $(200 \mu \mathrm{m})$ consisted of a Melinex O PET $(100 \mu \mathrm{m}) / 3 \mathrm{M}$ adhesive $(100 \mu \mathrm{m})$ film, custom designed using CorelDraw graphics suite X5 software (COREL OEM product) and machined using a 30-watt $\mathrm{CO}_{2}$ laser cutter (Epilog Zing Model 10000, USA). The Epilog Model 10000 system is a class 3R laser product (IEC 60825-1) and complies with 21 CFR 1040.10 and 1040.11 FDA regulations. Bonding of the insulation layer/film to the printed base layer resulted in an in-house designed sensor with a sealed thin-layer capillary (flow) channel of approximately $4 \mu \mathrm{L}$ capacity and $200 \mu \mathrm{m}$ height as shown in Fig. 1. These sensors are in effect single-use plastic strips/devices and their features include two indicator electrodes with a dimension of $1.5 \mathrm{~mm} \times 5 \mathrm{~mm}$ each and a spacing of $0.5 \mathrm{~mm}$ in between them. Finally, after fabrication, all sensors were stored dry at room temperature prior to use for electrochemical ELISA measurements.

\section{O. ostertagi optical ELISA}

The optical ELISA analysis was performed using the commercially available SVANOVIR ${ }^{\circledR} O$. ostertagi-Ab indirect ELISA kit according to the manufacturer's instructions. Briefly, samples $(100 \mu \mathrm{L})$ were incubated for $60 \mathrm{~min}$ in non-infectious

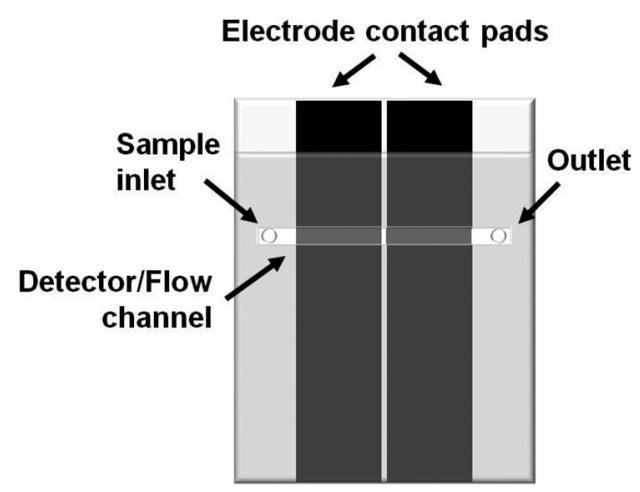

Fig. 1 Graphical illustration of a sensor (two indicator electrodes printed flow through device) used for the e-ELISA measurements. Detector channel dimensions: $15 \mathrm{~mm} \times 1.5 \mathrm{~mm}$; indicator electrode area dimensions (each): $5 \mathrm{~mm} \times 1.5 \mathrm{~mm}$. 
O. ostertagi antigen coated wells of microtitre strips. After washing $(\times 3$, PBS-T), O. ostertagi antibodies bound to the antigen were subsequently incubated with an anti-bovine IgG-HRP conjugate $(100 \mu \mathrm{L})$ for another $60 \mathrm{~min}$. Following the complex formation and washing $(\times 3$, PBS-T), the substrate ABTS solution (2,2'-azino-bis(3-ethylbenzothiazoline-6-sulphonic acid)) was added and incubated for $30 \mathrm{~min}$ and the reaction was stopped by adding SDS stop solution (sodium dodecyl sulfate). All incubations were carried out under ambient conditions (room temperature). Absorbance data were collected using a microplate reader (Synergy H1 Hybrid Multi-Mode Microplate Reader, BioTek ${ }^{\mathrm{TM}}$, Vermont, USA) at $405 \mathrm{~nm}$ via software (BioTek Gen5 ${ }^{\mathrm{TM}}$ 2.01). In the case of TMB, the results were read at $450 \mathrm{~nm}$ using $\mathrm{SDS}$ and $\mathrm{H}_{2} \mathrm{SO}_{4}$ stop solutions.

\section{Electrochemical ELISA (e-ELISA) measurements}

Chronocoulometry experiments were performed using the computer-controlled potentiostat $\mathrm{CHI660C}(\mathrm{CH}$ Instruments Inc., Austin, USA). The methodology involved on-plate ELISA steps (including wash stages, $\times 3$, PBS-T) followed by manual delivery of the reaction solution into the electrochemical flow cell/channel at the final assay step. A potential difference (50 $\mathrm{mV}$ ) was applied across the dual electrodes, following delivery of $4 \mu \mathrm{L}$ of the microwell final supernatant solution into the flow cell (detector/flow channel). The flow of charge resulting from the redox reactions, being dependent on the $\mathrm{TMB}_{\text {red }}: \mathrm{TMB}_{\mathrm{ox}}$ ratio, was measured over a period of 20 seconds and sampled at 10 seconds $\left(Q_{10}\right)$ for response comparison with the blank (PBS-T), negative and positive samples provided with the commercial kit. All experiments were carried out at ambient temperature of about $25^{\circ} \mathrm{C}$.

\section{Statistical analysis}

All results are expressed as the mean \pm SD. Statistical significance of differences was calculated using the standard twotailed $t$-test. Unless stated otherwise, data were collected from at least three independent experiments using three identical sensor devices. The level of probability for statistical significance was established at $p \leq 0.05$ for all analyses.

\section{Results and discussion}

\section{Principle of the proposed electrochemical immunoassay (e-ELISA)}

Herein, we present a strategy for electrochemically assaying the levels of $O$. ostertagi antibodies in serum samples using an antigen-indirect ELISA format. Scheme 1 illustrates the entire experimental setup of the proposed assay, which utilised the printed electrochemical sensor for measuring plate-bound $O$. ostertagi antibodies via the anti-bovine IgG-HRP-catalysed oxidation of TMB upon application of a constant potential and subsequent charge measurement (which depends on the $\left.\mathrm{TMB}_{\mathrm{red}} / \mathrm{TMB}_{\mathrm{ox}}\right)$. If $O$. ostertagi antibodies are present in a sample, they bind to the antigen coated wells and subsequently form a complex with the IgG-HRP conjugate. Upon TMB $/ \mathrm{H}_{2} \mathrm{O}_{2}$ addition, the HRP conjugate catalyses the $\mathrm{H}_{2} \mathrm{O}_{2}$-mediated oxidation of TMB, resulting in fluctuations of $\mathrm{TMB}_{\mathrm{ox}} / \mathrm{TMB}_{\text {red }}$ concentrations, measured via the charge $(\mu \mathrm{C})$ vs. time (s) response. The procedure links the peroxidase signalling reaction (which occurs off-chip) and the thin layer printed sensor function which responds to $\mathrm{TMB}_{\mathrm{ox}} / \mathrm{TMB}_{\text {red }}$ alterations, upon manual delivery of a microwell reagent into the flow through cell (detector/flow channel). Advantages of this off-chip format include isolation of the printed electrodes from immunoreagents and associated background interference effects.

The detection system utilised here is based on twin-electrode thin layer electrochemistry (small channel height, $200 \mu \mathrm{m}$, allows rapid electrochemical reaction) involving the application of a small potential difference $(50 \mathrm{mV})$ across two geometrically identical electrodes which measure changes in charge passed as a result of redox molecule concentrations. ${ }^{16-19}$ In peroxidase measurements the electro-

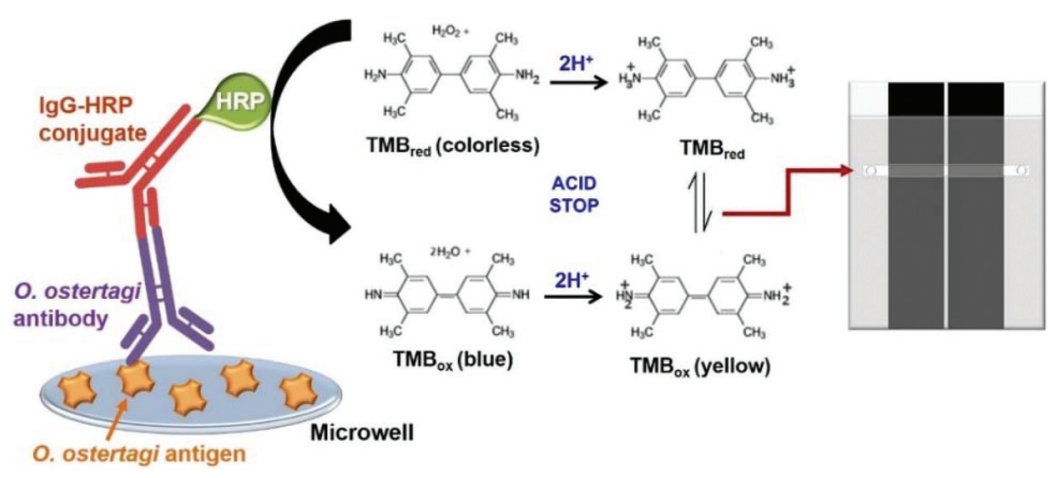

Scheme 1 Schematic representation of the indirect electrochemical ELISA format used for the detection of O. ostertagi infection. O. ostertagi antibodies bind to the antigen coated wells and subsequently form a complex with the HRP conjugated antibody. A potential difference (50 mV) applied across the two identical electrodes produces a flow of charge $\left(Q_{10}\right)$ as a result of the electrode reactions. The detection stage of the assay involves peroxidase oxidation of the TMB substrate by the HRP-conjugated antibody, oxidising the reduced colourless form of the redox molecule, $\mathrm{TMB}_{\text {red }}$ to a blue-coloured diimine molecule, $\mathrm{TMB}_{\mathrm{ox}}$ (oxidised form of $\mathrm{TMB}$ ). The indicator electrodes of the sensor respond to the shift in $\mathrm{TMB}_{\text {red }}-\mathrm{TMB}_{\mathrm{ox}}$ concentrations and in doing so monitor the progression of the peroxidase reaction (and hence $O$. ostertagi antibody levels). 
chemical charge accompanying the HRP driven conversion of $\mathrm{TMB}_{\text {red }}$ into $\mathrm{TMB}_{\mathrm{ox}}$ was monitored over the measurement period (20 seconds) with background subtraction. Electrochemical charge values were sampled at 10 seconds $\left(Q_{10}\right)$ from charge-time plots.

In other words, the detection stage of the assay involves peroxidase oxidation of the TMB substrate by the HRP-conjugated antibody, oxidising the reduced colourless form of the redox molecule, $\mathrm{TMB}_{\text {red }}$, to a blue-coloured diimine molecule, $\mathrm{TMB}_{\mathrm{ox}}$ (oxidised form of TMB). The indicator electrodes of the sensor respond to the shift in $\mathrm{TMB}_{\text {red }}-\mathrm{TMB}_{\mathrm{ox}}$ concentrations and in doing so monitor the progression of the peroxidase reaction and hence $O$. ostertagi antibody levels.

\section{Electrochemical assessment of ELISA reagents - enzymatic (HRP) oxidation of TMB}

The TMB electrochemical signal was monitored with and without $\mathrm{HRP}$ in the presence of $\mathrm{H}_{2} \mathrm{O}_{2}$. The activity was measured by chronocoulometry $(\Delta E=50 \mathrm{mV}$, vol $=4 \mu \mathrm{L}$, measurement time/pulse width $=20$ seconds, charge $(Q)$ sampling time $(t)=10$ seconds, $\left.Q_{10}\right)$. As expected, the electrochemical response $\left(Q_{10}\right)$ was higher when HRP was present (Fig. 2), with HRP/TMB response being 18.5-fold higher compared to TMB alone and 23.8-fold higher than that of the blank sample (PBS-T). The results show that the method can effectively detect HRP through the TMB redox activity.

The performance of the e-ELISA and electrochemical sensor was evaluated in response to positive and negative $O$. ostertagi serum samples, using fixed concentrations of the capture

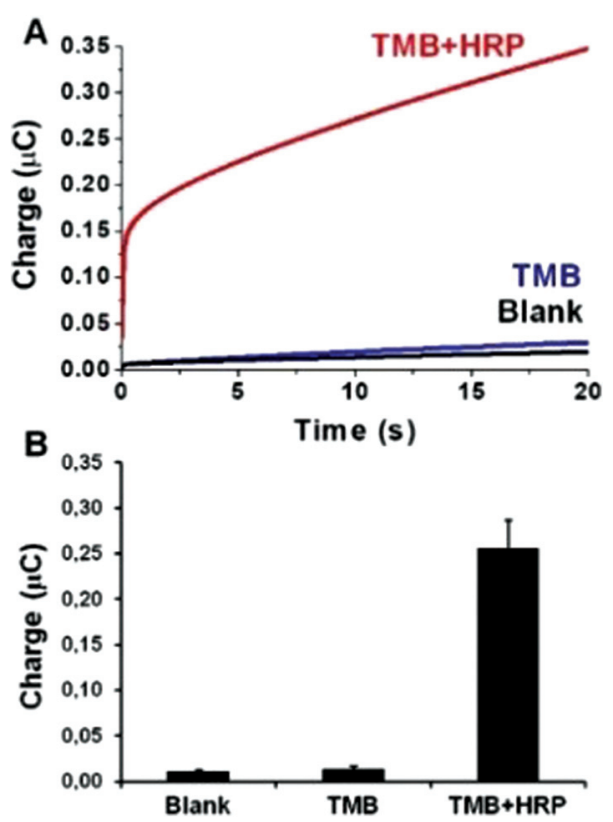

Fig. 2 (A) Representative chronocoulometric response curves of TMB with and without HRP along with the blank measurement (PBS-T). Applied potential $(\Delta E)=50 \mathrm{mV}$, volume $=4 \mu \mathrm{L}$, pulse width $=20 \mathrm{~s}$, charge $\left(Q_{10}\right)$ sampling time $=10 \mathrm{~s}$. (B) Column graph signals of charge response $\left(Q_{10}\right)$ corresponding to the chronocoulometric curves shown in $(A)$. antigen and HRP conjugate (SVANOVIR ${ }^{\circledR}$ Ostertagia-Ab ELISA kit) and varied redox mediators (ABTS vs. TMB), stop solutions (SDS vs. $\mathrm{H}_{2} \mathrm{SO}_{4}$ ) and incubation times (150 min vs. $70 \mathrm{~min} v s$. $25 \mathrm{~min})$.

The commercial optical ELISA kit uses ABTS as a chromogenic substrate whereas both ABTS and TMB were tested in the case of e-ELISA (sensor response) optimisations. HRP reaction stop solution SDS was recommended for the commercial kit, which was tested and compared against $\mathrm{H}_{2} \mathrm{SO}_{4}$. Incubation time analysis was performed, and total incubation time was reduced significantly from the recommended $150 \mathrm{~min}$ (for the commercial ELISA kit) to $25 \mathrm{~min}$. The e-ELISA sensor device was capable of successful differentiation of positive and negative $O$. ostertagi serum samples.

\section{Electrochemical ELISA (e-ELISA) and sensor performance analysis}

All experimental manipulations were assayed both spectrophotometrically and electrochemically. When performing the immunoassay according to the SVANOVIR kit instructions (ABTS as a substrate and SDS as a stop solution), the optical readings were found to significantly differentiate between $O$. ostertagi positive and negative serum samples, as expected (Fig. 3A). Electrochemical ELISA (e-ELISA) under similar conditions was capable of differentiating positive and negative serum samples (Fig. 3B); however the responses were not as notable as the ones observed in the optical ELISA. When calculating the positive to negative $\left(\mathrm{P} / \mathrm{N}_{150}\right)$ serum ratio for a total incubation time of $150 \mathrm{~min}$ for both assays (Fig. 5), the optical kit results produced the higher $\mathrm{P} / \mathrm{N}$ ratio $\left(\mathrm{P} / \mathrm{N}_{150}=3.7\right)$ compared to the electrochemical ELISA performance $\left(\mathrm{P} / \mathrm{N}_{150}=1.5\right)$. $\mathrm{P} / \mathrm{N}$ ratio was chosen as a figure of merit, since it is known to be an index value used in immunoassay optimisation studies,
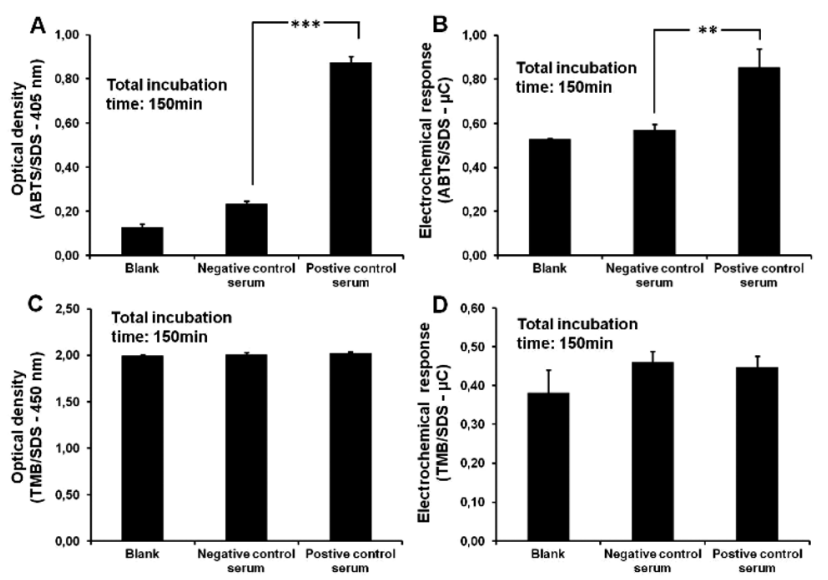

Fig. 3 (A) Optical and (B) electrochemical ELISA readings of positive and negative control serum samples using the SVANOVIR® Ostertagia-Ab ELISA kit (ABTS/SDS). (C) Optical and (D) electrochemical ELISA responses using the TMB substrate (to compare with ABTS used in the commercial kit) and SDS stop solution. Data represent mean \pm SD, twotailed $t$-test, ${ }^{* *} p \leq 0.01,{ }^{* \star *} p \leq 0.001$ vs. negative control serum. Total assay incubation time: $150 \mathrm{~min}$. 
where the experimental conditions that produce the maximum $\mathrm{P} / \mathrm{N}$ ratios are selected during assay development. ${ }^{20,21}$

Given TMB's wide application in electrochemical immunoassays, ${ }^{22}$ this redox substrate was considered as an alternative to ABTS (commercial kit) and evaluated together with the use
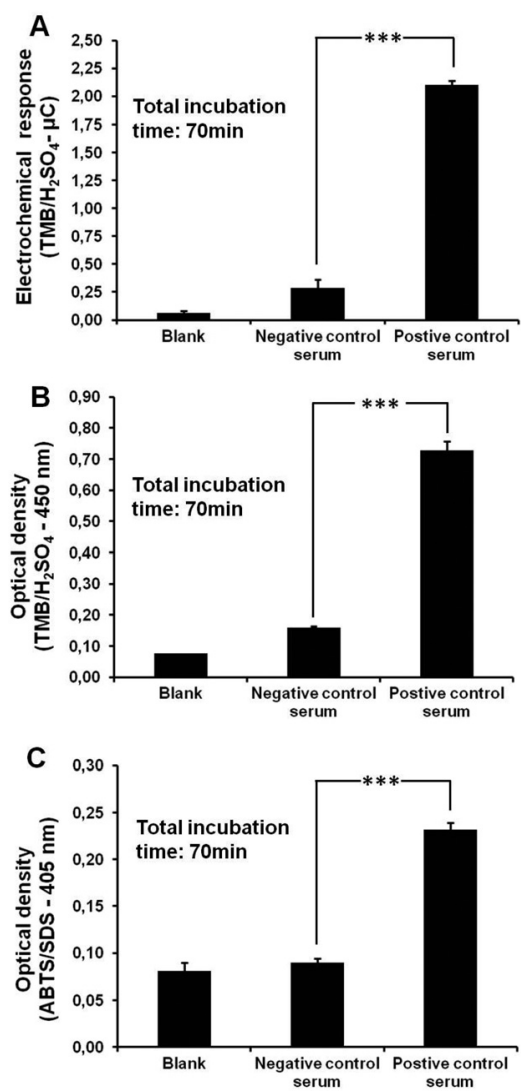

Fig. 4 (A) TMB electrochemical and (B) optical ELISA readings using $\mathrm{H}_{2} \mathrm{SO}_{4}$ as a stop solution with $70 \mathrm{~min}$ total incubation time. (C) ABTS/ SDS optical ELISA readings with $70 \mathrm{~min}$ total incubation time. Data represent mean $\pm S D$, two-tailed $t$-test, ${ }^{* *} p \leq 0.01,{ }^{* * *} p \leq 0.001$ vs. negative control serum. of SDS stop solution. However, both optical and electrochemical measurements using TMB/SDS combination were unable to differentiate between the blank, O. ostertagi positive and negative serum samples (Fig. 3C and D). This is also clearly evident from the positive to negative control ratios for the $\mathrm{TMB} / \mathrm{SDS}$ based optical $\left(\mathrm{P} / \mathrm{N}_{150}=1.01\right)$ and electrochemical $\left(\mathrm{P} / \mathrm{N}_{150}=0.98\right)$ ELISA approaches with a total incubation time of $150 \mathrm{~min}$ (Fig. 5). This gives an indication that the TMB system is not working well with SDS and the $\mathrm{pH}$ effect seems critical for TMB's optical and electrochemical interpretation. $\mathrm{TMB}$, in the presence of HRP and hydrogen peroxide, is converted into the $\mathrm{TMB}_{\mathrm{ox}}$ product, with an absorption maximum at $653 \mathrm{~nm}$. Acidification with $\mathrm{H}_{2} \mathrm{SO}_{4}(\mathrm{pH}<1)$ yields a stable diimine oxidation product (as shown in the reaction in Scheme 1) which has a maximum absorbance peak at $450 \mathrm{~nm}$, while it is also electroactive, thus allowing electrochemical detection. $^{23}$

For this reason, we opted for $\mathrm{H}_{2} \mathrm{SO}_{4}$ as the stop solution and TMB as the redox substrate for all consecutive experiments. As it can be seen in Fig. $4 \mathrm{~A}$ and $\mathrm{B}$, when switching to $\mathrm{TMB} / \mathrm{H}_{2} \mathrm{SO}_{4}$ combination, both optical and electrochemical measurements were able to significantly differentiate between $O$. ostertagi positive and negative serum samples, even when the total incubation time was reduced to $70 \mathrm{~min}$. The $\mathrm{P} / \mathrm{N}_{70}$ control serum ratios were 7.4 and 4.5 for the electrochemical and optical readings respectively (Fig. 5), suggesting that the electrochemical $\mathrm{TMB} / \mathrm{H}_{2} \mathrm{SO}_{4}$ detection approach is more sensitive than its optical counterpart in the $70 \mathrm{~min}$ incubation time-frame.

Additional optical readings with ABTS and SDS in the 70 min incubation time (Fig. 4C) also produced satisfactory sample differentiation, with lower absorbance values than the original kit instructions (Fig. 3A) and lower $\mathrm{P} / \mathrm{N}$ ratio $\left(\mathrm{P} / \mathrm{N}_{150}=\right.$ 3.7 vs. $\mathrm{P} / \mathrm{N}_{70}=2.6$, Fig. 5). Comparison of the $\mathrm{P} / \mathrm{N}$ ratios of the commercially available optical ELISA (ABTS/SDS) with those of the electrochemical ELISA $\left(\mathrm{TMB} / \mathrm{H}_{2} \mathrm{SO}_{4}\right)$ shows almost 2.9 times better performance for e-ELISA in a reduced $70 \mathrm{~min}$ incubation time-frame, i.e. $\mathrm{P} / \mathrm{N}_{70}=7.4$ for e-ELISA $\left(\mathrm{TMB} / \mathrm{H}_{2} \mathrm{SO}_{4}\right)$ and $\mathrm{P} / \mathrm{N}_{70}=2.6$ for commercial optical ELISA (ABTS/SDS).

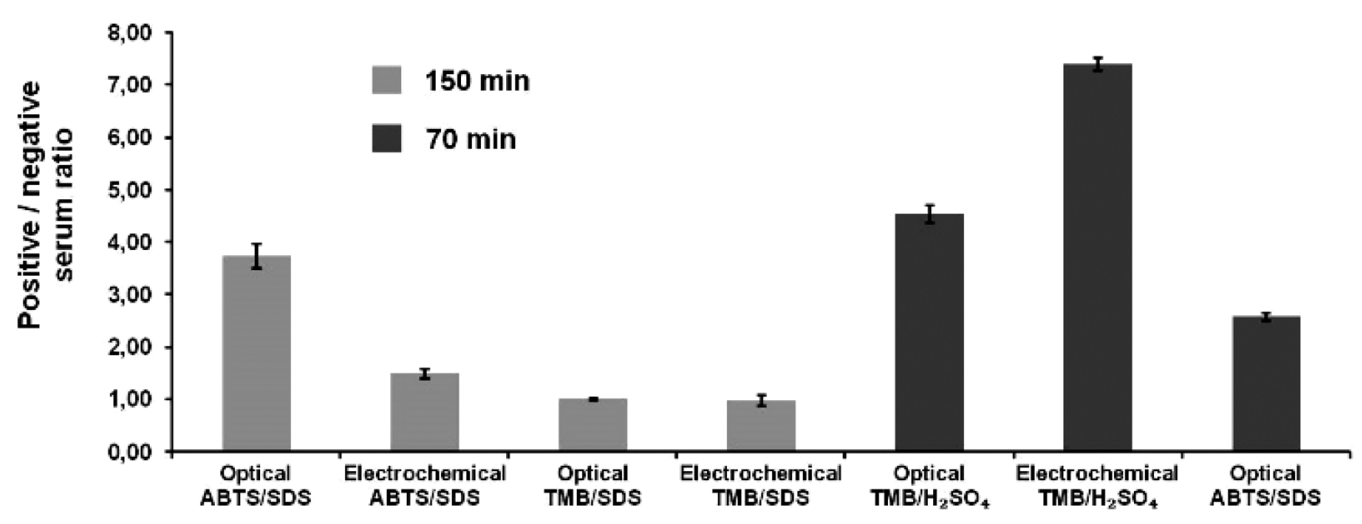

Fig. 5 Positive to negative control ratios $(\mathrm{P} / \mathrm{N})$ of the studied optical and electrochemical ELISA approaches for $150 \mathrm{~min}$ and $70 \mathrm{~min}$ total incubation times. 

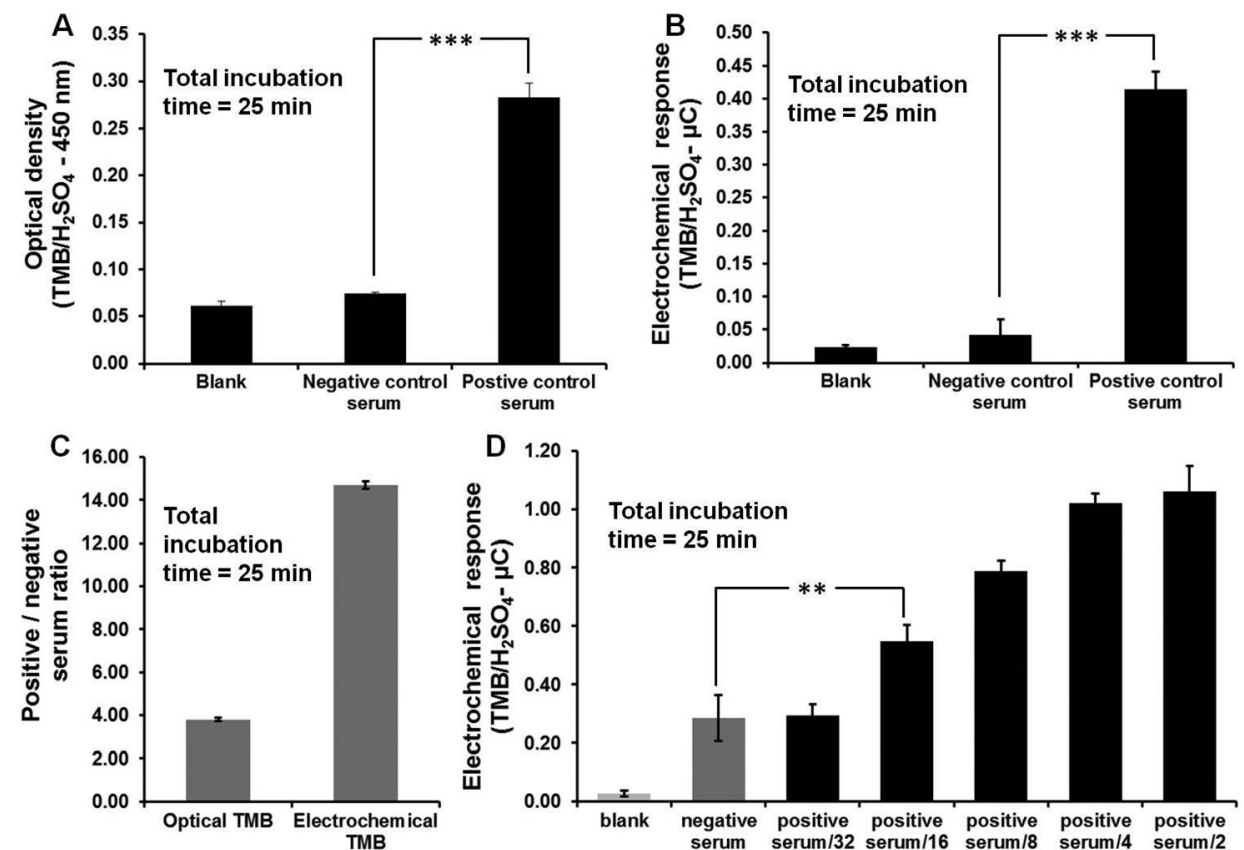

Fig. 6 Reduced incubation time ( $25 \mathrm{~min}$ ) immunoassays with both (A) optical and (B) electrochemical ELISA approaches using TMB (substrate) and $\mathrm{H}_{2} \mathrm{SO}_{4}$ (stop solution). (C) Positive to negative control serum ratios for (A) and (B). (D) Electrochemical readings of 2-fold serial dilutions of positive control serum. Data represent mean $\pm S D$, two-tailed $t$-test, ${ }^{* *} p \leq 0.01,{ }^{* *} p \leq 0.001$ vs. negative serum.

To further evaluate the e-ELISA performance $\left(\mathrm{TMB} / \mathrm{H}_{2} \mathrm{SO}_{4}\right.$ based electrochemical immunoassay), total incubation time (sample + enzyme conjugate + substrate incubations) was reduced to $25 \mathrm{~min}$ and both optical and electrochemical ELISA measurements were recorded (Fig. 6A and B). Both assays were able to give statistically significant differences for positive and negative control serums, with the $\mathrm{P} / \mathrm{N}_{25}$ ratio of the electrochemical assay being 14.7 (Fig. 6C), 3.9-fold higher than the one for the optical immunoassay $\left(\mathrm{P} / \mathrm{N}_{25}=3.8\right)$. Shorter incubation period $(25 \mathrm{~min})$ resulted in a better performance which may be attributed to the appropriate peroxidase reaction time and substrate incubation period, compared to the longer incubations where the conjugate adsorption could easily affect the ELISA/sensor performance. In future, the output of the sensor needs to be fully characterised for a large number of high and low analyte concentrations to avoid any false assignment.

Since the e-ELISA $\left(\mathrm{TMB} / \mathrm{H}_{2} \mathrm{SO}_{4}\right.$ based electrochemical sensor approach) was able to perform efficiently under the reduced 25 min assay time, we opted to evaluate it against a series of diluted positive control serum samples. As it can be seen in Fig. 6D, the electrochemical immunoassay performed well even at the 16 -fold dilution of the positive serum, giving a statistically significant difference from the negative control serum ( $p \leq 0.01$; two-tailed $t$-test), even after such low incubation periods ( 25 minutes). This increased sensitivity demonstrates the potential of the sensor-based e-ELISA as a rapid and sensitive detection tool for the analysis of the parasitic nematode Ostertagia ostertagi (antibodies) in serum samples. This also suggests the potential future quantitative capabilities of the sensing technique in Ostertagia ostertagi analysis in serum or milk samples.

\section{Conclusions}

A simple approach for the development of an electrochemical ELISA based on in-house fabricated sensors for the sensitive and accelerated detection of parasitic nematode Ostertagia ostertagi antibodies in serum samples is presented. Costeffective and disposable sensors were designed and produced using cheap polyester and chemical adhesive materials, stencil printing and laser machining techniques. The electrochemical approach (TMB $/ \mathrm{H}_{2} \mathrm{SO}_{4}$ based e-ELISA) successfully differentiated between positive and negative control serum samples, with a P/N control ratio 1.6 times that of optical ELISA (TMB/ $\mathrm{H}_{2} \mathrm{SO}_{4}$ combination) and 2.9 times that of the commercial ELISA kit (optical, ABTS/SDS combination). Furthermore, the e-ELISA approach is quicker and required only $25 \mathrm{~min}$ (total assay incubation time) with even better response (approximately 4 -fold higher) than that of the optical immunoassay under similar conditions. The e-ELISA is specific (highly selective $\mathrm{Ab}-\mathrm{Ag}$ interactions) and capable of detecting up to 16-fold dilutions of a positive control serum sample in 25 min of total incubation period (compared to $150 \mathrm{~min}$ recommended for the commercial ELISA kit) which demonstrates its potential as a rapid and highly sensitive screening tool/format for Ostertagia ostertagi analysis. Such printed sensors are scalable (low cost materials), require low power (applied potentials, $\mathrm{mV}$ ) and generate faradaic charge responses of the $\mathrm{nC} / \mu \mathrm{C}$ level.

Future work will target the advancement of e-ELISA (immunosensor development) and sensor characterisation by performing analysis in real samples and collecting larger data sets for reliability, reproducibility and quantification information with an ultimate target for diagnostic testing of para- 
sitic O. ostertagi infections. Prior work in our group has established portable electrochemical ELISA formats for infectious diseases and has concluded that irreversible physisorption of peroxidase and peroxidase-antibody conjugates occurs rapidly at carbon surfaces. Advancement to a portable Ostertagia ostertagi device for on-field applications demands overcoming the nonspecific binding interactions (on-chip), performing reagent stability analysis, and integration of microfluidics for reagent system delivery with a sensor/detector and a signal display/readout unit.

\section{Conflicts of interest}

There are no conflicts to declare.

\section{Acknowledgements}

Dr Baljit Singh and Prof. Eithne Dempsey thank and acknowledge Enterprise Ireland and European Regional Development Fund for provision of financial support under the Commercialisation Fund (CF/2011/1053A: Ireland's EU Structural Funds Programmes 2007-2013, co-funded by the Irish Government and the European Union). MiCRA Biodiagnostics at Technological University Dublin (TU Dublin - Tallaght Campus) is funded and supported under the Enterprise Ireland Technology Gateway Programme.

\section{Notes and references}

1 M. T. Fox, Vet. Parasitol., 1997, 72, 285-308.

2 S. J. LeBlanc, K. D. Lissemore, D. F. Kelton, T. F. Duffield and K. E. Leslie, J. Dairy Sci., 2006, 89, 1267-1279.

3 L. Rinaldi, G. Hendrickx, G. Cringoli, A. Biggeri, E. Ducheyne, D. Catelan, E. Morgan, D. Williams, J. Charlier, G. von Samson-Himmelstjerna and J. Vercruysse, Geospat. Health, 2015, 9, 257-259.

4 J. A. Van Wyk and E. Mayhew, Onderstepoort J. Vet. Res., 2013, 80, 539, DOI: 10.4102/ojvr.v80i1.539.

5 J. Vercruysse and E. Claerebout, Vet. Parasitol., 2001, 98, 195-214.

6 M. Sekiya, A. Zintl and M. L. Doherty, Ir. Vet. J., 2013, 66, 14, DOI: 10.1186/2046-0481-66-14.
7 J. Sanchez, I. R. Dohoo, F. Markham, K. Leslie and G. Conboy, Vet. Parasitol., 2002, 109, 75-90.

8 A. Warsinke, A. Benkert and F. W. Scheller, Fresenius. J. Anal. Chem., 2000, 366, 622-634.

9 F. Davis and S. Higson, in Immunoassays: Development, Applications and Future Trends, Jenny Stanford Publishing, 2017, pp. 291-326.

10 K. Yamanaka, M. C. Vestergaard and E. Tamiya, Sensors, 2016, 16(10), 1761, DOI: 10.3390/s16101761.

11 F. S. Felix, A. L. B. Baccaro and L. Angnes, Sensors, 2018, 18(12), 4124, DOI: 10.3390/s18124124.

12 K. K. Mistry, K. Layek, A. Mahapatra, C. RoyChaudhuri and H. Saha, Analyst, 2014, 139, 2289-2311, DOI: 10.1039/ C3AN02050A.

13 G. Hughes, K. Westmacott, K. C. Honeychurch, A. Crew, R. M. Pemberton and J. P. Hart, Biosensors, 2016, 6(4), 50, DOI: $10.3390 /$ bios6040050.

14 V. K. Rao, M. K. Sharma, P. Pandey and K. Sekhar, World J. Microbiol. Biotechnol., 2006, 22, 1135-1143.

15 F. Ricci, G. Adornetto and G. Palleschi, Electrochim. Acta, 2012, 84, 74-83.

$16 \mathrm{H}$. L. Kies, Thesis - Theorie en toepassingen van titraties volgens de methode van het dead-stop eindpunt, Delft University of Technology, December 1956. http:// resolver.tudelft.nl/uuid:1fce0290-9099-400f-ab66-c12541746094.

17 K. G. Stone and H. G. Scholten, Anal. Chem., 1952, 24, 671674.

18 T. Loughman, B. Singh, B. Seddon, P. Noone and S. Padmanabhan, Analyst, 2017, 142, 2725-2734.

19 P. Kissinger and W. R. Heineman, Laboratory Techniques in Electroanalytical Chemistry, CRC Press, 2nd edn, 1996, 1008 pp. (ISBN 9780824794453 ).

20 G. R. Zhang, J. Y. Zeng, Y. M. Zhu, S. J. Dong, S. Zhu, R. S. Yu, C. Duoji, Z. H. Lei and Z. Li, Intervirology, 2011, $55,12-20$.

21 M. A. Bakheit, L. Schnittger, D. A. Salih, K. Boguslawski, D. Beyer, M. Fadl and J. S. Ahmed, Parasitol. Res., 2004, 92, 299-302.

22 F. Ricci, G. Adornetto and G. Palleschi, Electrochim. Acta, 2012, 84, 74-83.

23 P. Fanjul-Bolado, M. B. González-García and A. CostaGarcía, in Analytical and Bioanalytical Chemistry, SpringerVerlag, 2005, vol. 382, pp. 297-302. 